Check for updates

Cite this: RSC Adv., 2018, 8, 38529

Received 5th August 2018

Accepted 27th October 2018

DOI: $10.1039 / c 8 r a 06575 f$

rsc.li/rsc-advances

\section{Chinese liquor extract enhances inflammation resistance in RAW 264.7 and reduces aging in Caenorhabditis elegans}

\author{
Jie Liu, ab Huailing Wang, ${ }^{\text {ab }}$ Xiaoyu Liu, ${ }^{\text {ab }}$ Guohao Zhang, ${ }^{\mathrm{b}}$ Pingchang Yang ${ }^{\text {ab }}$ \\ and Zhigang Liu (iD *ab
}

\begin{abstract}
Recent reports have indicated that the ingredients in Chinese liquor possess multiple bioactivities. The objective of this study was to evaluate the potential effects of Chinese liquor extract (CME) on the resistance to inflammation in mononuclear macrophages (RAW 264.7 cell line) and aging in Caenorhabditis elegans (C. elegans). The results showed that CME suppressed key lipopolysaccharide (LPS)-induced pro-inflammatory mediators, tumor necrosis factor- $\alpha$, and nitric oxide in vitro. Furthermore, CME inhibited activation of mitogen-activated protein kinase (MAPK) and phosphatidylinositol-3 kinase (PI3K)/AKT pathways in LPS-stimulated cells. Further studies also showed that CME improved stress resistance of nematodes under infection conditions. Moreover, CME increased the expression of immune-related genes, such as lys-7. Based on these results, our findings provide mechanistic insights about the protection provided by CME against LPS-induced inflammation in RAW 264.7 cells, namely, inhibition of MAPK and PI3K/AKT pathways, as well as its capability against Pseudomonas aeruginosa- and Staphylococcus aureus-induced aging in C. elegans.
\end{abstract}

\section{Introduction}

Components of the innate immunity include neutrophils and macrophages, and their role is to initiate an inflammatory response, form phagocytes to kill pathogens, recruit natural killer cells, and facilitate the maturation and migration of dendritic cells that will initiate the adaptive immune response. ${ }^{1}$ Inflammation, which is accompanied by fatal damages, such as oxidative damage, may worsen numerous diseases. However, inflammation is not typically a negative phenomenon: it is the response of the immune system to the invasion of viruses or bacteria and other pathogens. Emerging evidence suggests that inflammations are related to immunosenescence and reciprocally, controlling the inflammatory status may allow a better chance of controlling immunosenescence. ${ }^{2}$ Furthermore, inflammation is an important and necessary part of the normal host responses to pathogens, but the overproduction of inflammatory molecules might also cause immune-related inflammatory diseases and eventually death. Therefore, low responders involved in regulation of innate defense mechanisms might better control inflammatory responses and immunosenescence. ${ }^{3}$

${ }^{a}$ Department of Allergy, The Third Affiliated Hospital of Shenzhen University, Shenzhen, 518020, China. E-mail: lzgszuniversity@sina.com; Fax: +86-075586671907; Tel: +86-0755-86671907

${ }^{b}$ The Research Center of Allergy \& Immunology, Shenzhen University School of Medicine, Shenzhen, 518060, China
Lipopolysaccharide (LPS), a pathogenic endotoxin that can induce inflammatory responses and oxidative stress, is widely used for evaluating anti-inflammatory efficacy. In particular, macrophages produce excess pro-inflammatory cytokines, tumor necrosis factor- $\alpha$ (TNF- $\alpha$ ), and nitric oxide (NO) when exposed to inflammatory stimuli, such as LPS. ${ }^{1}$ Accumulating research shows that LPS causes overproduction of proinflammatory mediators and cytokines by activating nuclear factor $-\kappa \mathrm{B}(\mathrm{NF}-\kappa \mathrm{B})$, which is associated with mitogen-activated protein kinase (MAPK) and phosphatidylinositol-3 kinase (PI3K)/AKT pathways. $^{2}$ In addition, inflammatory stress increases the production of reactive oxygen species (ROS) and reduces antioxidant enzymes., ${ }^{3,4}$

Caenorhabditis elegans (C. elegans) is a commonly used model for aging as it exhibits the properties of a short lifespan, simple anatomy, and easy feeding. ${ }^{5}$ In addition, C. elegans share many highly conserved biochemical pathways with humans. ${ }^{6}$ Pseudomonas aeruginosa (P. aeruginosa) and Staphylococcus aureus ( $S$. aureus), two common pathogens in humans, are capable of causing a diverse range of diseases from superficial skin infections and soft tissue abscesses to life-threatening infections such as sepsis, endocarditis, pneumonia, and toxic shock syndrome. ${ }^{7}$ Moreover, bacterial infections shorten the lifespan of nematodes and cause immunosenescence. ${ }^{8}$

Aging is a process accompanied by deterioration of tissues, organs, and organism as well as the debasement of immunity, adaptability, and anti-infection ability, and finally the termination of life. ${ }^{9}$ There is a reciprocal relationship between aging 
and immune function degradation. Therefore, anti-aging treatments can improve immune function, while improving immune function can delay aging. ${ }^{10}$ Previous reports have shown that intake of antioxidants, such as natural products and herbal formulations, could enhance anti-infection ability and extend the lifespan of C. elegans. ${ }^{11}$

Chinese liquor is an alcoholic beverage obtained from grains by complex fermentation processes. Research studied the aroma compounds in Moutai liquor with comprehensive twodimensional gas chromatography/time-of-flight mass spectrometry, and the results showed that a total of 528 components were identified in a Moutai liquor sample, including organic acids, alcohols, esters, ketones, aldehydes, acetals, lactones, and nitrogen-containing and sulfur-containing compounds, in addition to alcohol and water. ${ }^{4}$ Some studies have shown that Moutai liquor does not cause liver injury, but rather strengthen lipid peroxidation in the liver, induce the increase in metallothioneins (MT), inhibit the proliferation of hepatic stellate cell (HSC) and generation of collagen and enhance the effect of antioxidation owing to multiple bioactive substances present in it. ${ }^{5}$ Chinese liquor has historically been regarded as the most important Chinese medicine by herbalist doctors. ${ }^{\mathbf{1 2}}$

Recent reports have indicated that some substances in Chinese liquor, such as geraniol, possess a variety of bioactivities such as anticancer, anti-inflammatory, and antibacterial properties. ${ }^{13}$ Chinese liquor has been consumed in China for thousands of years, and numerous studies have reported that some ingredients in Chinese liquor have antiseptic, antiinflammatory, and antioxidant effects; however, no previous study has focused on the anti-infection effect of Chinese liquor. As infection-related morbidity and mortality is closely associated with environmental stress in a given population, potential stress-resistance effects of CME in C. elegans were also investigated in this paper. Evaluating the protective effect of CME and its underlying mechanisms will broaden the understanding of Chinese liquor and provide a reference for healthy drinking.

\section{Materials and methods}

\section{Chemicals and reagents}

Chinese liquor (53\% v/v) was obtained from KweiChow Moutai Distillery in Guizhou province, China. Geraniol (Ger), lipopolysaccharide (LPS), 2',7'-dichlorofluorescin diacetate (DCFHDA), 5-fluoro-20-deoxyuridine (FUDR), and Paraquat were purchased from Sigma-Aldrich (St. Louis, MO, USA). All antibodies were purchased from Cell Signaling Technology (Danvers, MA, USA). The RAW 264.7 cells were obtained from the Cell Bank of the Chinese Academy of Sciences (Shanghai, China).

\section{Chinese liquor extract preparation}

Chinese liquor extract (CME) samples were prepared by the following method. Initially, $50 \mathrm{~mL}$ Chinese liquor, $20 \mathrm{~mL} \mathrm{H}_{2} \mathrm{O}$, and $90 \mathrm{~mL} \mathrm{CHCl}_{3}$ were added to a $250 \mathrm{~mL}$ separating funnel. The $\mathrm{CHCl}_{3}$ layer was evaporated at $0{ }^{\circ} \mathrm{C}$ using a Turbovap Sample Concentrator. Approximately $0.39 \mathrm{mg}$ of the residue remained and was taken as $\mathrm{CME}$ and stored at $-20{ }^{\circ} \mathrm{C}$ in the dark. Mentions of brand names do not imply any research contact with the liquor manufacturer nor are these mentions for advertising purposes.

\section{Cell and nematode culture}

RAW 264.7 cells were maintained in Dulbecco's Modified Eagle's Medium containing $10 \%(\mathrm{v} / \mathrm{v})$ fetal bovine serum, Lglutamine $(2 \mathrm{mM})$, penicillin $\left(100 \mathrm{U} \mathrm{mL}^{-1}\right)$ and streptomycin $\left(100 \mathrm{U} \mathrm{mL}^{-1}\right)$ at $37{ }^{\circ} \mathrm{C}$ in a humidified atmosphere containing $5 \% \mathrm{CO}_{2}$; culture medium was changed every 2 days. Cells in logarithmic growth phase were selected for experiments and divided into the following six experimental treatment groups: control, LPS, ethanol + LPS, Mou + LPS, CME + LPS, and Ger + LPS. Nematodes were cultured at $25{ }^{\circ} \mathrm{C}$ on nematode growth medium (NGM) plates and seeded with Escherichia coli OP50. Lyosgeny broth and slow-killing medium were prepared according to previously reported methods. ${ }^{\mathbf{1 4}}$

\section{Determination of intracellular ROS and NO in RAW 264.7 cells}

The cytotoxic activity of CME was detected using a previously described method, ${ }^{15}$ while intracellular ROS production was detected with a DCFH-DA using another previously reported method. ${ }^{16}$ Briefly, RAW 264.7 cells were pre-treated with chemicals for $24 \mathrm{~h}$, and then stimulated with LPS $\left(100 \mu \mathrm{g} \mathrm{mL}{ }^{-1}\right)$. After incubation for $24 \mathrm{~h}$, the cells were stained with $10 \mu \mathrm{M}$ DCFH-DA at $37^{\circ} \mathrm{C}$ in the dark. After $0.5 \mathrm{~h}$, the cells were washed with phosphate-buffered saline (PBS) twice, and then placed in an Ascent FL fluorescence plate reader $\left(37^{\circ} \mathrm{C}\right)$. Emission at $535 \mathrm{~nm}$ was measured after excitation at $485 \mathrm{~nm}$ for $20 \mathrm{~min}$. For NO concentration detection, cells were cultured using the same method before collecting cell supernatants. NO concentration was detected using a commercial NO ELISA kit (Beyotime, Shanghai, China) according to the manufacturer's instructions.

\section{Western blot analysis}

RAW 264.7 cells were incubated with chemicals [geraniol, CME, Chinese liquor (53\%), ethanol (v : v/ethanol : $\left.\mathrm{H}_{2} \mathrm{O} / 53: 47\right)$ ] for $24 \mathrm{~h}$ prior, and then stimulated with LPS $\left(100 \mu \mathrm{g} \mathrm{mL} \mathrm{m}^{-1}\right)$ for $24 \mathrm{~h}$. After incubation, the cells were collected, washed with PBS, lysed in $100 \mathrm{~mL}$ lysis buffer containing protease and phosphatase inhibitor cocktails, and centrifuged at $13000 \times g$ for $15 \mathrm{~min}$. An immunoblotting assay was performed as previously reported using $\beta$-actin protein as a measurement of the amount of protein analyzed. ${ }^{17}$

\section{Nematode lifespan assays}

Nematodes were maintained on NGM plates and seeded with Escherichia coli OP50 at $25{ }^{\circ} \mathrm{C}$ to obtain L4-larvae nematodes, as previously reported. ${ }^{18}$ Young adult worms were transferred to NGM plates containing FUDR $(50 \mu \mathrm{M})$ to prevent the growth of progeny. This transfer day was designated as day 0. For the safety assessment, CME, geraniol, Chinese liquor and ethanol were added to NGM plates $(100 \mu \mathrm{L}$ per plate) at concentrations ranging from $0-0.40 \mathrm{mg} \mathrm{mL}^{-1}$ for $48 \mathrm{~h}$ before detecting effects 
on lethality, growth, and movement behavior using previously reported methods. ${ }^{19}$

\section{Anti-Pseudomonas aeruginosa and anti-Staphylococcus aureus infection assays}

Experiments were performed according to a previously reported method with some modifications. ${ }^{20}$ The minimal inhibitory concentration (MIC) of CME, geraniol, Chinese liquor and ethanol against $P$. aeruginosa and $S$. aureus was detected using a previously reported method. ${ }^{21}$ Briefly, young adult worms were transferred to new NGM plates containing FUDR $(50 \mu \mathrm{M})$ to prevent the growth of progeny, and then pretreated for $48 \mathrm{~h}$ in the following groups: blank (Con); ethanol (V:V/ethanol : $\left.\mathrm{H}_{2} \mathrm{O} / 53: 47\right)$ (Eth); Chinese liquor (53\%) (Mou); CME (CME); and geraniol (Ger). Synchronized young adult nematodes were cultured in NGM plates with or without chemicals for 24,48 , or $72 \mathrm{~h}$. After incubation, approximately 80 nematodes were randomly picked and placed into plates coated with $P$. aeruginosa or $S$. aureus, which were cultured at $25^{\circ} \mathrm{C}$; this day was considered as day 0 . Each day, the number of surviving nematodes was counted until all nematodes were dead. Triplicate plates were used for each group. Nematodes that showed no movement on touching gently were considered dead.

\section{Real time-polymerase chain reaction (RT-PCR) assays}

Nematodes were treated as described above. After pretreatment with CME for $48 \mathrm{~h}$ and infection with $P$. aeruginosa or $S$. aureus for $48 \mathrm{~h}$, total RNA was isolated from about 1500 nematodes using Trizol reagent. ${ }^{22}$ Primer sequences for RT-PCR are listed in Table 1 and results are reported as mean $\pm \mathrm{SD}$.

\section{Results and discussion}

\section{Cytotoxicity of CME on RAW 264.7 cells}

After obtaining the CME, we analyzed it with GC-MS, and the results showed that the CME is mainly composed of esters, polyols, acids, aromatic compounds, furans, aldehydes and pyrazines. HPLC analysis showed that the concentration of geraniol in Chinese liquor is $1.107 \pm 0.013 \mu \mathrm{g} \mathrm{L} \mathrm{L}^{-1}(p>0.05)$.

In consideration of the fact that compounds may themselves elicit cytotoxicity, the effect of each chemical on RAW 264.7 cell viability was tested. The results showed that no chemical tested (maximum concentration $50.0 \mu \mathrm{g} \mathrm{mL}^{-1}$ ) had a significant effect

Table 1 Primer sequence of RT-PCR

\begin{tabular}{ll}
\hline Gene & Primer sequence \\
\hline lys-7 & F: $5^{\prime}$-GTCAAGGTTCCCCCGATTGT-3' \\
& R: $5^{\prime}$-ATCCTTGTCCTGCTGGGTTG-3' \\
clec-60 & F: $5^{\prime}$-ATAGGTTGTGGCGTATGGGC-3' \\
& R: $5^{\prime}$-AATGTTCAATCGGCCACCCT-3' \\
fo8g5.6 & F: $5^{\prime}$-TGTCCCACTGTCACAAGCTC-3' \\
& R: $5^{\prime}$-TCGGGAATTGGGTTCGACC-3'
\end{tabular}
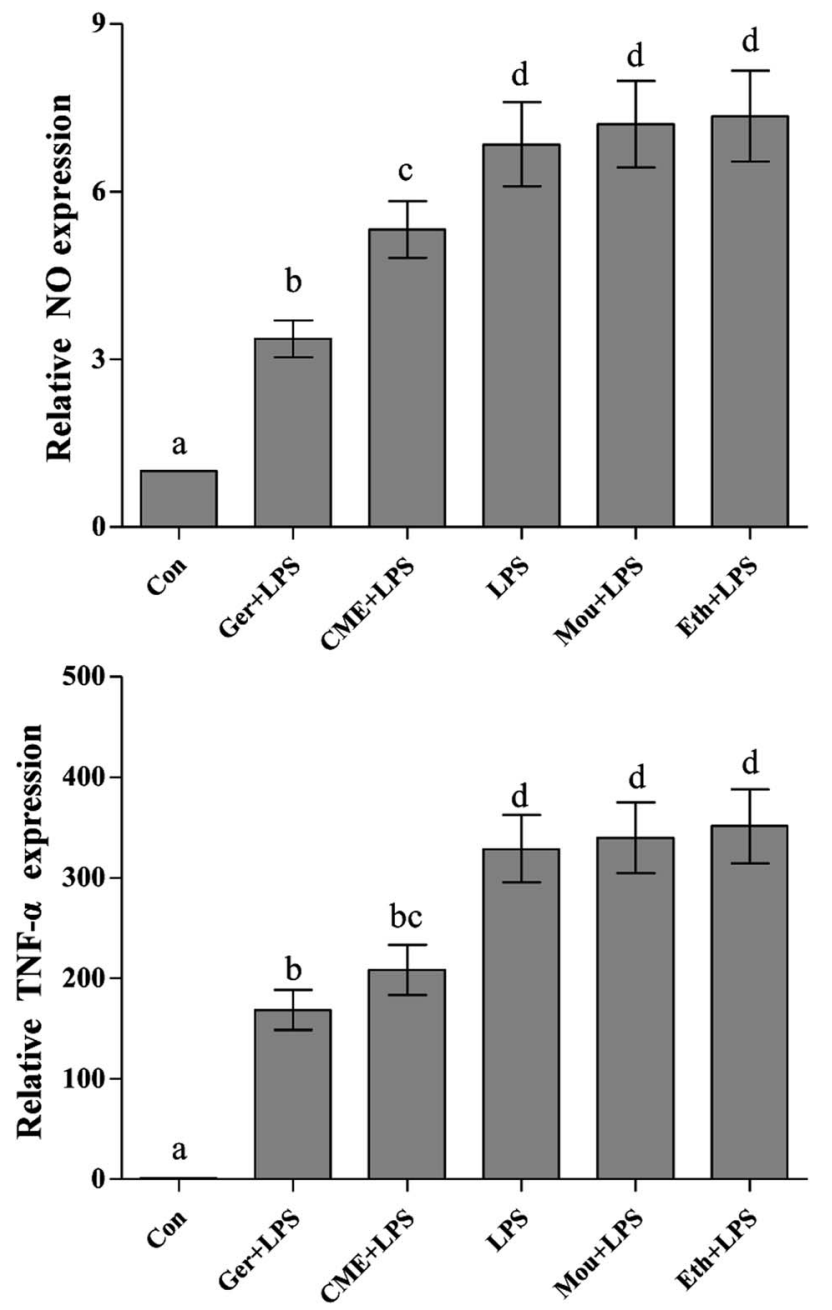

Fig. 1 CME represses LPS-induced $\mathrm{NO}$ and TNF- $\alpha$ production. RAW264.7 cells were pretreated with the indicated reagents for $24 \mathrm{~h}$, and then stimulated with LPS for $24 \mathrm{~h}$. NO and TNF- $\alpha$ levels were measured as described in the Materials and methods. (Con): DMEM only; (Ger + LPS): geraniol at $50.0 \mu \mathrm{g} \mathrm{mL}^{-1}$ for $24 \mathrm{~h}$ and then with LPS at $100.0 \mu \mathrm{g} \mathrm{mL}^{-1}$ for $24 \mathrm{~h}$; (CME + LPS): CME at $50.0 \mu \mathrm{g} \mathrm{mL}^{-1}$ for $24 \mathrm{~h}$ and then with LPS at $100.0 \mu \mathrm{g} \mathrm{mL}^{-1}$ for $24 \mathrm{~h}$; (LPS): LPS at $100.0 \mu \mathrm{g}$ $\mathrm{mL}^{-1}$ for $24 \mathrm{~h}$; (Mou + LPS): Chinese liquor (53\%) at $50.0 \mu \mathrm{g} \mathrm{mL}^{-1}$ for $24 \mathrm{~h}$ and then with LPS at $100.0 \mu \mathrm{g} \mathrm{mL}^{-1}$ for $24 \mathrm{~h}$; (Eth + LPS): ethanol (V : V/ethanol : $\mathrm{H}_{2} \mathrm{O} / 53$ : 47) at $50.0 \mu \mathrm{g} \mathrm{mL}^{-1}$ for $24 \mathrm{~h}$ and then with LPS at $100.0 \mu \mathrm{g} \mathrm{mL}^{-1}$ for $24 \mathrm{~h}$. Data are represented as the mean $\pm \mathrm{SD}$ $(n=3)$. Bars with different letters indicate significant difference between groups $(p<0.05)$.

on RAW 264.7 cell viability. Accordingly, the anti-inflammatory effects of CME on RAW 264.7 cells were detected at $50.0 \mu \mathrm{g}$ $\mathrm{mL}^{-1}$.

\section{Effects of CME on pro-inflammatory mediators}

NO, a representative pro-inflammatory mediator, is regulated by inducible NO synthase (iNOS). ${ }^{23}$ In addition, major proinflammatory cytokines, such as TNF- $\alpha$, are overexpressed in macrophages stimulated by LPS, where they contribute to the pathogenesis of various inflammatory diseases. ${ }^{24}$ After treatment with LPS, uncontrolled activation of RAW 264.7 cells can 


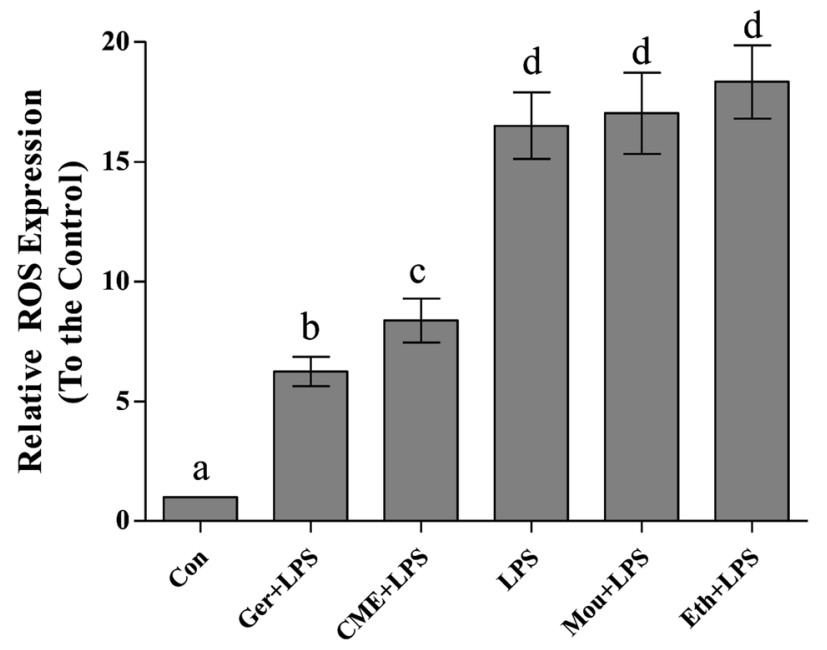

Fig. 2 CME represses LPS-induced ROS production. RAW264.7 cells were pretreated with the indicated reagents for $24 \mathrm{~h}$, and then stimulated with LPS for $24 \mathrm{~h}$. ROS production was measured as described in the Materials and methods. (Con): DMEM only; (Ger + LPS): geraniol at $50.0 \mu \mathrm{g} \mathrm{mL} \mathrm{L}^{-1}$ for $24 \mathrm{~h}$ and then with LPS at $100.0 \mu \mathrm{g} \mathrm{mL}^{-1}$ for $24 \mathrm{~h}$; (CME + LPS): CME at $50.0 \mu \mathrm{g} \mathrm{mL}^{-1}$ for $24 \mathrm{~h}$ and then with LPS at 100.0 $\mu \mathrm{g} \mathrm{mL}^{-1}$ for $24 \mathrm{~h}$; (LPS): LPS at $100.0 \mu \mathrm{g} \mathrm{mL}^{-1}$ for $24 \mathrm{~h}$; (Mou + LPS): Chinese liquor (53\%) at $50.0 \mu \mathrm{g} \mathrm{mL} \mathrm{L}^{-1}$ for $24 \mathrm{~h}$ and then with LPS at $100.0 \mu \mathrm{g} \mathrm{mL}^{-1}$ for $24 \mathrm{~h}$; (Eth + LPS): ethanol (V: V/ethanol: $\mathrm{H}_{2} \mathrm{O}$ / $53: 47$ ) at $50.0 \mu \mathrm{g} \mathrm{mL}^{-1}$ for $24 \mathrm{~h}$ and then with LPS at $100.0 \mu \mathrm{g} \mathrm{mL}^{-1}$ for $24 \mathrm{~h}$. Data are represented as the mean \pm SD $(n=3)$. Bars with different letters indicate significant difference between groups $(p<$ 0.05).

cause injury via overproduction of the inflammatory mediators such as NO and TNF- $\alpha,{ }^{25}$ which is consistent with the results presented in Fig. 1 (NO and TNF- $\alpha$ content in the control group was set as 1). These results indicated that LPS stimulation increased the NO concentration $(6.85 \pm 0.75)$ compared with the control group, but was significantly reduced by CME (5.32 \pm 0.51 ). In addition, TNF- $\alpha$ was significantly reduced on CME pretreatment $(208.3 \pm 25.2)$ compared with that observed in the LPS group $(328.9 \pm 33.4)$. These results suggested that CME played a significant role in modulating inflammatory cytokine expression. Geraniol showed a similar effect to CME: it reduced the concentration of NO by $50.8 \%$ and inhibited TNF- $\alpha$ protein expression by $48.7 \%$ compared with that of the LPSadministered group. Compared with the LPS group, the relative NO concentrations and TNF- $\alpha$ protein expressions in Chinese liquor and ethanol groups increased, but showed no significant difference.

\section{CME reduced ROS levels}

Inflammation and oxidative stress are now recognized as two important factors contributing to the development of many diseases, and ROS plays crucial roles in both processes. ${ }^{26}$ Indeed, oxidative stressors would cause serious damage to lipids, proteins, and DNA. ${ }^{27,28}$ Intake of red wine can naturally interrupt ROS expression and inhibit the progression of inflammation and oxidative stress. ${ }^{29}$ As shown in Fig. 2, relative ROS expression in the LPS group $(16.5 \pm 1.38)$ significantly

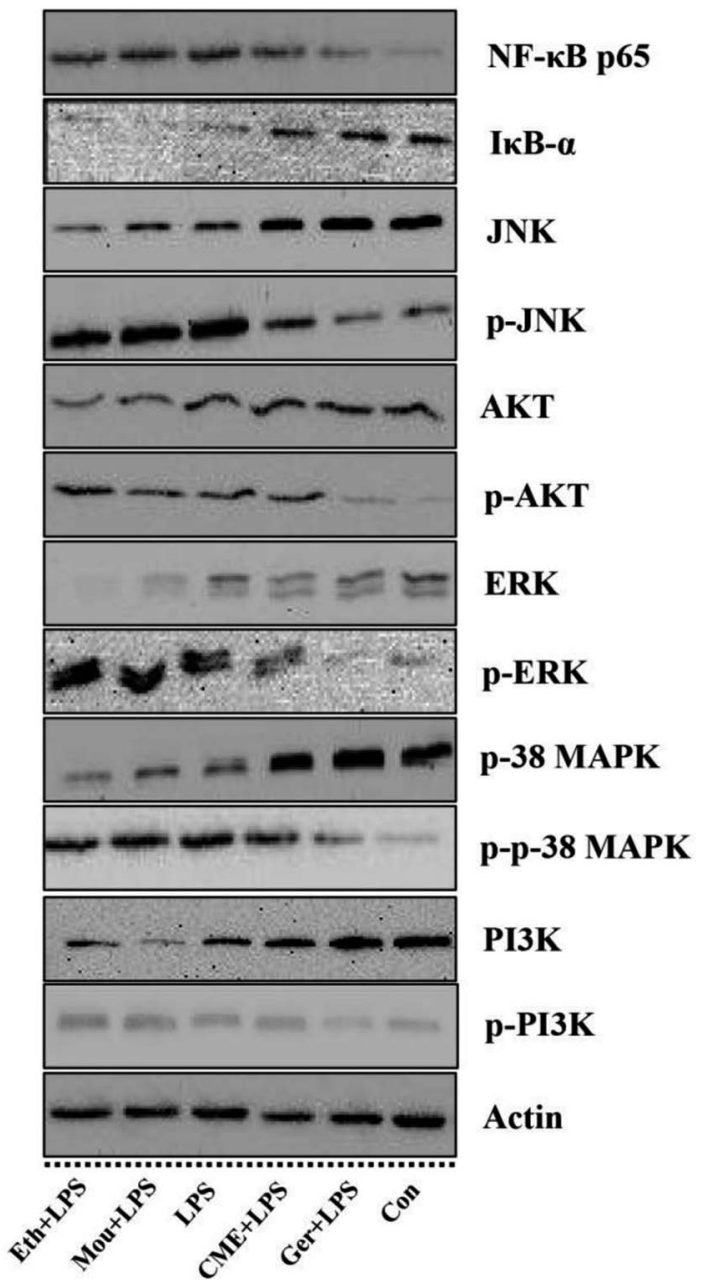

Fig. 3 The relative expression level of proteins including: NF- $\kappa B$ p65, I $\mathrm{B}-\alpha$, JNK, p38 MAPK, ERK, PI3K, AKT, p-JNK, p-p38 MAPK, p-ERK, pPI3K, p-AKT, and proteins in mononuclear macrophage RAW264.7 cells. Protein assay results were confirmed by western blotting, and the relative expression levels of the proteins were determined. (Con): DMEM only; (Ger + LPS): geraniol at $50.0 \mu \mathrm{g} \mathrm{mL}^{-1}$ for $24 \mathrm{~h}$ and then with LPS at $100.0 \mu \mathrm{g} \mathrm{mL}^{-1}$ for $24 \mathrm{~h}$; (CME + LPS): CME at $50.0 \mu \mathrm{g} \mathrm{mL}^{-1}$ for $24 \mathrm{~h}$ and then with LPS at $100.0 \mu \mathrm{g} \mathrm{mL} \mathrm{m}^{-1}$ for $24 \mathrm{~h}$; (LPS): LPS at $100.0 \mu \mathrm{g} \mathrm{mL}^{-1}$ for $24 \mathrm{~h}$; (Mou + LPS): Chinese liquor (53\%) at $50.0 \mu \mathrm{g}$ $\mathrm{mL}^{-1}$ for $24 \mathrm{~h}$ and then with LPS at $100.0 \mu \mathrm{g} \mathrm{mL}{ }^{-1}$ for $24 \mathrm{~h}$; (Eth + LPS): ethanol ( $V$ : V/ethanol: $\mathrm{H}_{2} \mathrm{O} / 53$ : 47) at $50.0 \mu \mathrm{g} \mathrm{mL}^{-1}$ for $24 \mathrm{~h}$ and then with LPS at $100.0 \mu \mathrm{g} \mathrm{mL}^{-1}$ for $24 \mathrm{~h}$. Bars with different letters mean significant difference between groups $(p<0.05)$.

increased compared with that in the control group. Pretreatment with CME or geraniol significantly reduced LPSinduced ROS expression $(8.37 \pm 0.91$ and $6.25 \pm 0.62$, respectively). In contrast to $\mathrm{CME}$ and geraniol, pre-treatment with ethanol or Chinese liquor increased relative ROS expression to $18.3 \pm 1.52$ and $17.0 \pm 1.69$, respectively. As the results showed that CME and geraniol reduced ROS expression, we inferred this effect may be associated with its antioxidant activity. However, neither ethanol nor Chinese liquor showed protective effects and instead increased the level of ROS. We inferred that the main component, ethanol, elicited these harmful effects and induced ROS expression. 

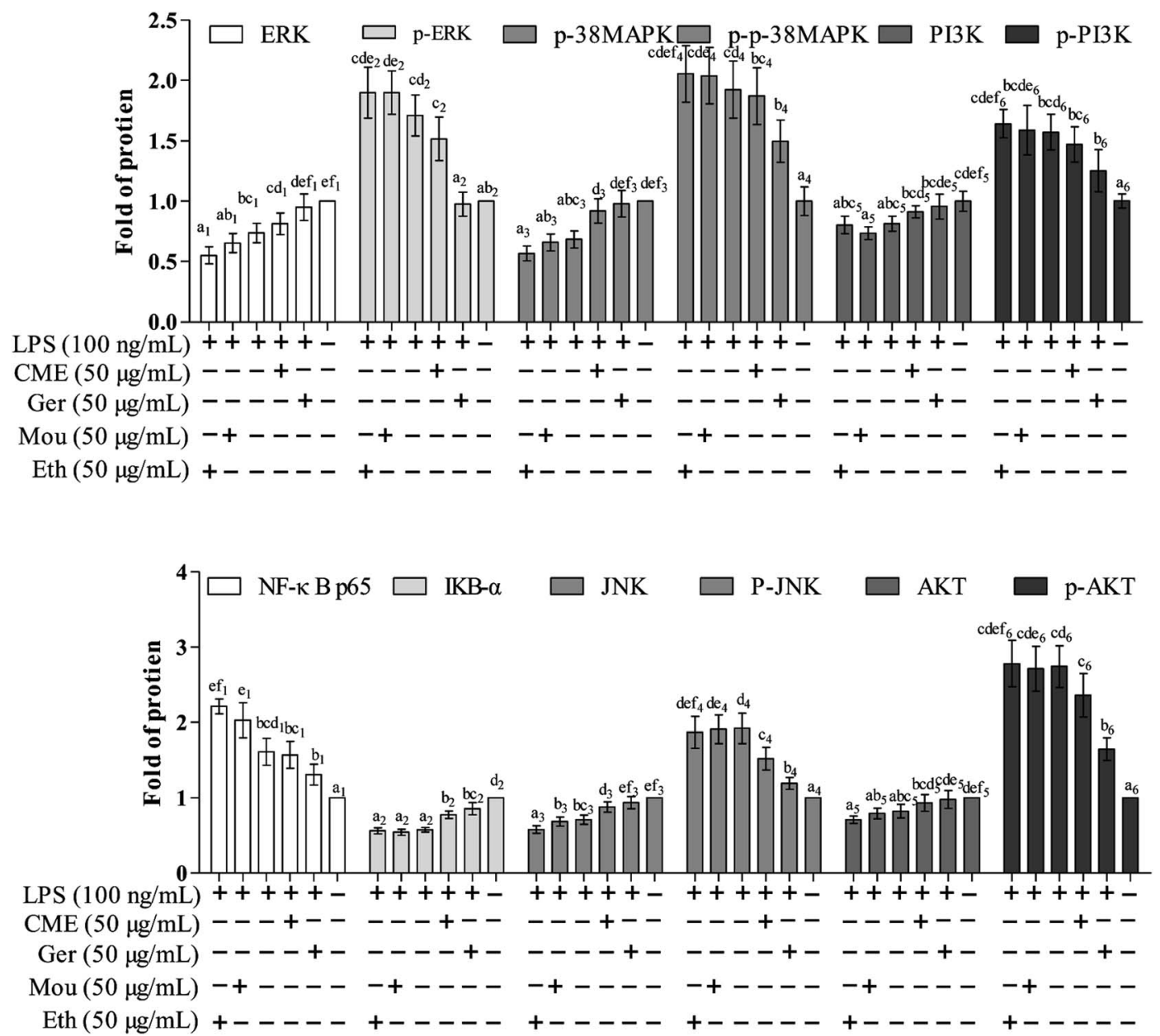

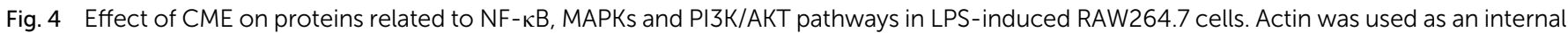
control. RAW264.7 cells were treated with indicated reagents. (Con): DMEM only; (Ger + LPS): geraniol at $50.0 \mu \mathrm{g} \mathrm{mL} \mathrm{m}^{-1}$ for $24 \mathrm{~h}$ and then with LPS at $100.0 \mu \mathrm{g} \mathrm{mL}^{-1}$ for $24 \mathrm{~h}$; (CME + LPS): CME at $50.0 \mu \mathrm{g} \mathrm{mL}^{-1}$ for $24 \mathrm{~h}$ and then with LPS at $100.0 \mu \mathrm{gL}^{-1}$ for $24 \mathrm{~h}$; (LPS): LPS at $100.0 \mu \mathrm{g} \mathrm{mL}{ }^{-1}$ for $24 \mathrm{~h}$; (Mou + LPS): Chinese liquor (53\%) at $50.0 \mu \mathrm{g} \mathrm{mL}^{-1}$ for $24 \mathrm{~h}$ and then with LPS at $100.0 \mu \mathrm{g} \mathrm{mL} \mathrm{L}^{-1}$ for $24 \mathrm{~h}$; (Eth + LPS): ethanol (V: V/ ethanol : $\mathrm{H}_{2} \mathrm{O} / 53$ : 47) at $50.0 \mu \mathrm{g} \mathrm{mL}^{-1}$ for $24 \mathrm{~h}$ and then with LPS at $100.0 \mu \mathrm{g} \mathrm{mL}^{-1}$ for $24 \mathrm{~h}$. The proteins expression levels were measured using western blot. The density of each lane was presented as mean \pm SD for at least three individual experiments. Blots were quantified using Image $J$ software. Bars with different letters indicate significant difference between groups $(p<0.05)$.

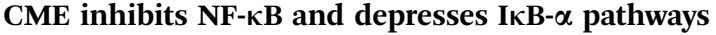

The NF- $\kappa \mathrm{B}$ signaling pathway is important for inflammatory responses. Activation of $\mathrm{NF}-\kappa \mathrm{B}$ requires phosphorylation of upstream I $\mathrm{KB}$ kinase (IKK), which contains two catalytic subunits, IKK $\alpha$ and IKK $\beta .^{30}$ Upon stimulation with LPS or proinflammatory cytokines, IKK is phosphorylated and activated by TGF- $\beta$-activated kinase 1 (TAK1), resulting in further phosphorylation and depression of $\mathrm{I} \kappa \mathrm{B}$ in the ubiquitination pathway. Subsequently, $\mathrm{NF}-\kappa \mathrm{B}$ releases from the $\mathrm{I} \kappa \mathrm{B} / \mathrm{NF}-\kappa \mathrm{B}$ dimer and translocates from the cytoplasm into the nucleus, inducing further pro-inflammatory gene expression and inflammatory responses. Therefore, inhibition of the NF- $\kappa B$ pathway may have a potential therapeutic effect. As such, the effect of CME on NF- $\kappa$ B pathway inhibition was investigated. As shown in Fig. 3 and 4, LPS stimulation markedly increased NF$\kappa \mathrm{B}$ p65 expression by $61.0 \%$ and reduced $\mathrm{I} \kappa \mathrm{B}-\alpha$ expression by $42.7 \%$ compared with those in the control group. However, with
CME pre-treatment, NF- $\kappa$ B p65 expression was markedly inhibited and $\mathrm{I} \kappa \mathrm{B}-\alpha$ expression was upregulated. Compared with the LPS group, expression of NF- $\kappa \mathrm{B}$ p65 was reduced by $11.8 \%$ and that of $\mathrm{I} \kappa \mathrm{B}-\alpha$ was increased by $35.1 \%$. These results suggest that CME may act as a negative regulator of NF- $\kappa \mathrm{B}$ activation. However, ethanol and Chinese liquor showed contrasting effects as they increased NF- $\kappa \mathrm{B}$ p65 expression and reduced $\mathrm{I} \kappa \mathrm{B}-\alpha$ expression compared with those in the LPS group. We inferred that the ethanol in these groups played the main functions.

\section{CME inhibits activation of MAPK and PI3K/AKT pathways}

MAPKs play significant roles against inflammation, and extracellular signal-regulated protein kinase (ERK) can regulate, at least in part, NO production, iNOS expression, and TNF$\alpha$ secretion. $^{31}$ Moreover, rapid and lasting activation of c-Jun $\mathrm{NH}(2)$-terminal kinase MAPK (JNK) can be induced by LPS in 


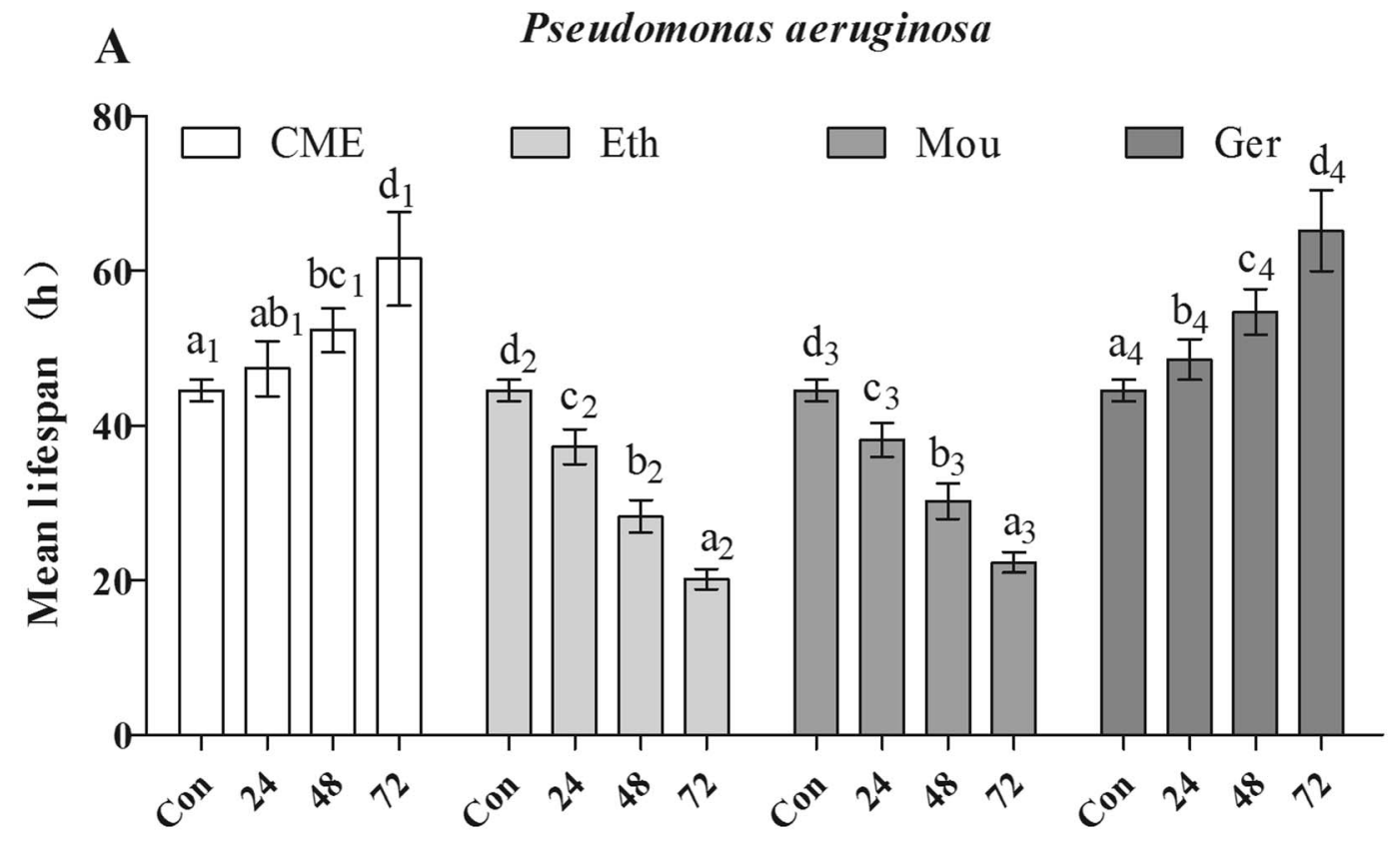

Groups

B Staphylococcus aureus

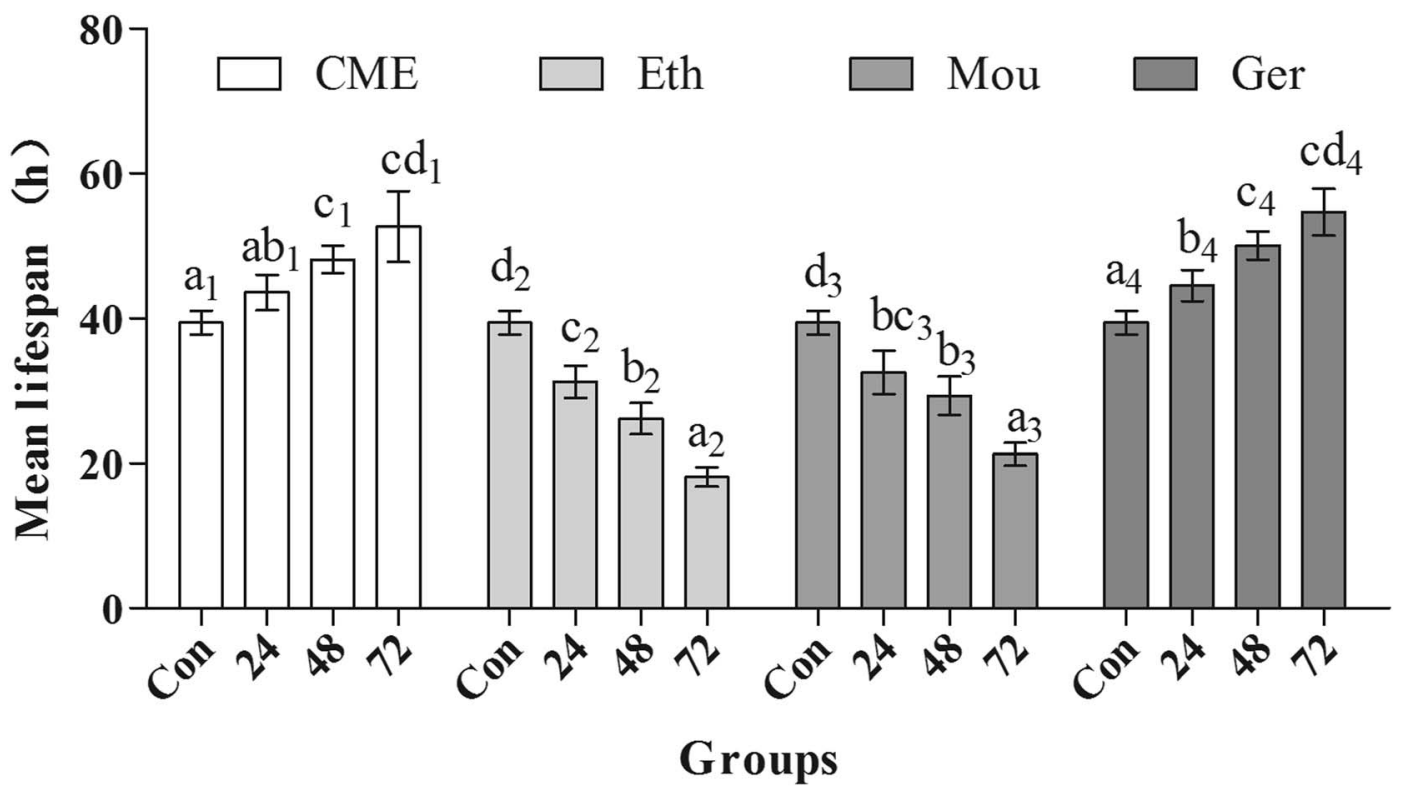

Fig. 5 Effect of CME treatment on anti-aging in C. elegans. The young adult nematodes were treated with CME for 24,48 and $72 \mathrm{~h}$, then exposed to Pseudomonas aeruginosa (A) and Staphylococcus aureus (B) and monitored the survivals every hour ( $N \geq 80$ animals). The experiment was repeated multiple times and a representative trial is shown. Bars with different letters indicate significant difference between groups $(p<0.05)$.

RAW 264.7 cells, and inhibition of JNK reduces LPS-induced TNF- $\alpha$ activity. A previous study has also shown that the PI3K/ AKT signaling pathway is involved in inflammation. ${ }^{32}$ As shown in Fig. 3 and 4, LPS stimulation promoted the phosphorylation of JNK, p38 MAPK, ERK, as well as PI3K and AKT by approximately $92.5 \%, 92.1 \%, 71.3 \%, 57.1 \%$, and $174 \%$, respectively, compared with those in the control group. Moreover, LPS inhibited the expression of JNK, p38 MAPK, ERK,
PI3K, and AKT. Furthermore, with CME treatment, JNK, p38 MAPK, ERK, PI3K, and AKT expression increased, and their phosphorylation was inhibited compared with LPS groups. Thus, we concluded that CME may suppress the inflammatory response by inhibiting MAPK and PI3K/AKT pathways. However, both ethanol and Chinese liquor showed contrasting effects to CME, and played pro-inflammatory roles in response to LPS. 


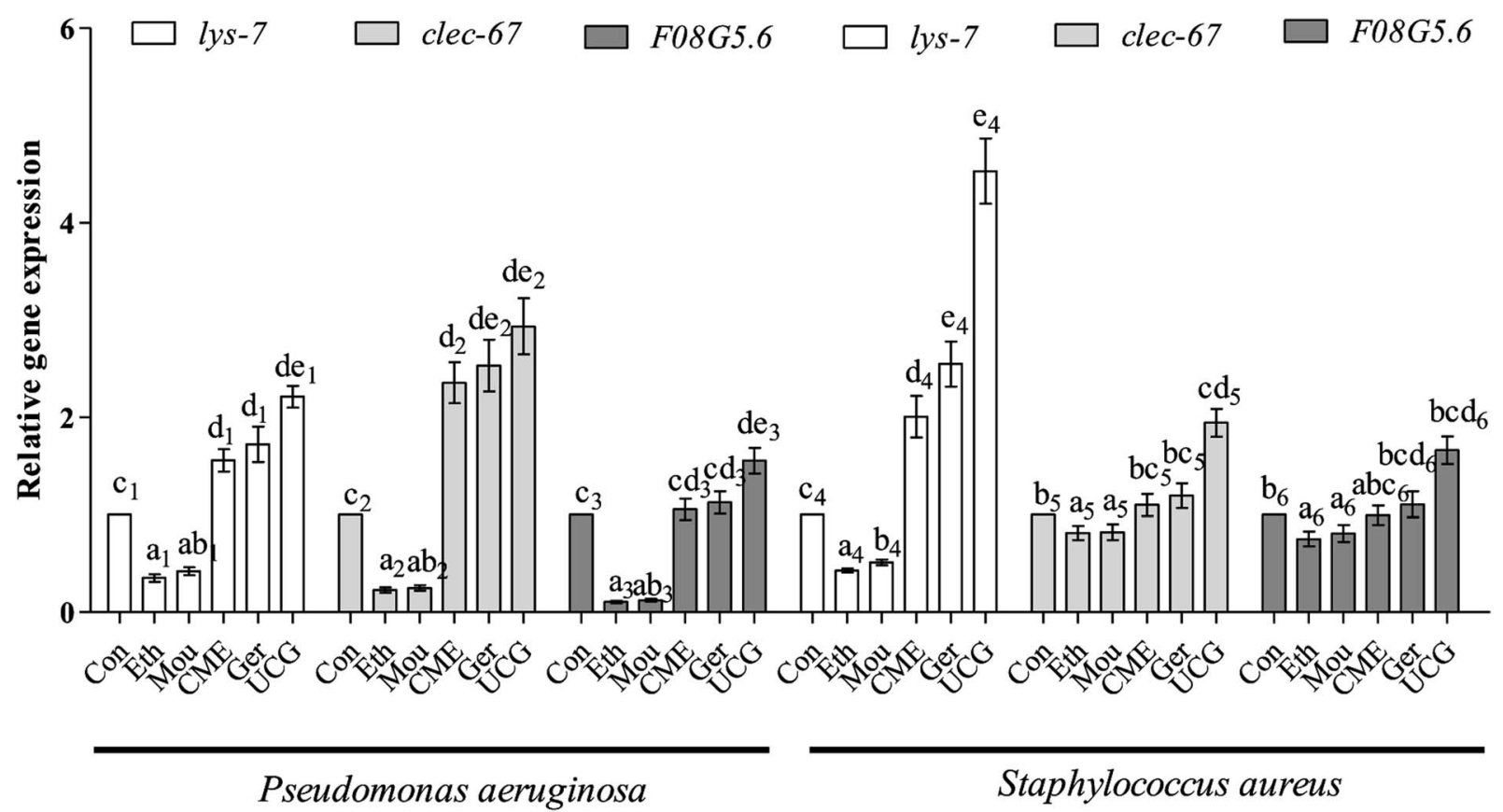

Fig. 6 The relative expression level of mRNA including: lys-7, clec-60, F08G5.6 in C. elegans. Assay results were confirmed by RT-PCR, and the relative expression levels of the mRNA were determined. Bars with different letters indicate significant difference between groups $(p<0.05)$.

\section{CME enhanced the anti-infection ability of nematodes}

C. elegans is a popular animal model for aging studies because it has a short lifespan, rapid generation, and experimental flexibility. In addition, $C$. elegans shares highly conserved biochemical pathways and many similar aging aspects with humans. From the initial identification of single gene mutations capable of increasing aging to the insulin-like growth factor signaling pathway, molecular mechanisms of aging have gradually become an increased focus of research. C. elegans has become the major model organism contributing to the discovery of molecular mechanisms of aging.

As previously reported, antioxidants increase the lifespan of nematodes, and some non-ethanol ingredients in Chinese liquor, such as geraniol, exhibit good antioxidant activity in vitro. ${ }^{33}$ Resveratrol, a polyphenol antioxidant found in red wine, has been the subject of intense interest in recent years for a range of unique anti-aging properties. ${ }^{34}$ Blueberry wine with proanthocyanidin also exhibited optimal anti-aging effects on C. elegans. $^{35}$ Clinically, alcohol is used for sterilization. In general, the substances in Chinese liquor may inhibit bacterial growth, thus affecting the lifespan of nematodes. Therefore, we tested the MIC of CME, geraniol, Chinese liquor and ethanol, to avoid this hypothesis. We found that treatment with $0-0.40 \mathrm{mg}$ $\mathrm{mL}^{-1}$ of CME, geraniol, Chinese liquor and ethanol did not affect the growth of bacteria or development, brood size, movement behavior, or viability of nematodes. Thus, a concentration of $0.20 \mathrm{mg} \mathrm{mL} \mathrm{m}^{-1} \mathrm{CME}$, geraniol, Chinese liquor and ethanol were selected for subsequent studies.

After pre-treatment with CME for 24,48 , or $72 \mathrm{~h}$, lifespans were greatly improved, as shown in Fig. 5. Mean lifespans in control groups were $44.6 \pm 1.40 \mathrm{~h}$ and $39.5 \pm 1.60 \mathrm{~h}$ after infection with $P$. aeruginosa or $S$. aureus, respectively. However, with CME pre-treatment, mean lifespans in infected groups increased. With CME pre-treatment for 24,48 , or $72 \mathrm{~h}$, mean lifespans were $47.4 \pm 3.60 \mathrm{~h}, 52.3 \pm 2.80 \mathrm{~h}$, and $61.6 \pm 6.00 \mathrm{~h}$ in $P$. aeruginosa-infected groups, respectively; compared with the control group, the mean lifespans increased by $6.37 \%, 17.4 \%$, and $38.2 \%$, respectively. In addition, the mean lifespans of nematodes in the $S$. aureus-infected groups were also enhanced to $43.6 \pm 2.50 \mathrm{~h}, 48.2 \pm 1.90 \mathrm{~h}$, and $52.7 \pm 4.90 \mathrm{~h}$ with CME pretreatment for 24,48 , and $72 \mathrm{~h}$ respectively; compared with the control group, the lifespans increased by $10.5 \%, 22.0 \%$, and $33.5 \%$, respectively. For the ethanol and Chinese liquor groups, the lifespans of nematodes did not extend, but instead shortened. We inferred that the presence of ethanol may play a harmful effect and weakened the anti-stress ability of nematodes.

\section{Genetic requirements for increased anti-infection with CME treatment}

Bacteria are capable of causing disease and infection, which can abbreviate the lifespan of nematodes and cause aging. Expression levels of immune and aging effector genes significantly changed upon bacterial infection. During the initial stage of infection, C-Type lectin genes (e.g., clec-60) appear to be responsible for enhanced adhesion and antibacterial activity. ${ }^{36}$ The lysozyme gene lys-7, a presumptive antimicrobial gene critical for the induction of immune response, is upregulated in response to bacterial infection. Regulation of lys-7 mRNA against bacterial infection gradually increases with time of exposure and upregulation. ${ }^{37}$ The F08G5.6 gene belongs to a family of CUB-like proteins. Previous reports support the role 
for proteins carrying CUB-like domains in immunity against pathogens in C. elegans. The F08G5.6 gene is regulated by daf-2/ daf-16 and p38 MAPK pathways. ${ }^{38}$ After pre-treatment with CME for $48 \mathrm{~h}$, nematodes were infected with $P$. aeruginosa or $S$. aureus for $48 \mathrm{~h}$. Expression levels of immune-related genes were then tested by RT-PCR. The results are shown in Fig. 6 . For the $P$. aeruginosa infection condition, relative expression of lys-7 and clec-60 were upregulated 1.56- and 2.36-fold, respectively, in the $\mathrm{CME}$ treatment group compared with that in the control group. However, F08G5.6 expression was not different from the CME treatment group (1.05-fold).

For $S$. aureus infection conditions, relative expression of lys-7 was upregulated (2.00-fold) in the CME treatment group compared with that in the control group. However, expression of F08G5.6 and clec-60 were not different in the CME treatment groups (1.10- and 0.99-fold). Notably, geraniol showed better protective effect than CME, but both ethanol and Chinese liquor showed contrary effects, where the relative expression of immune-related genes lys-7, clec-60, and F08G5.6 was downregulated compared with that of the control group. Expression of immune genes is closely related to the anti-infection ability of C. elegans. The present study showed that CME upregulated the relative expression of lys-7 during $P$. aeruginosa and $S$. aureus infections. Thus, we inferred that the immune gene lys-7 played a role in stress resistance against bacterial infection. However, according to the results listed in Fig. 6, the relative expressions of lys-7, clec-60, and F08G5.6 in the uninfected control groups (UCG) were higher than those in the CME and geraniol groups. We inferred that treatments can attenuate but not offset the harm of bacteria.

\section{Conclusions}

Chinese liquor, one of the famous distilled liquors, is consumed worldwide and has components with diverse bioactivities. CME suppressed LPS-induced production of the key pro-inflammatory mediators NO and TNF- $\alpha$ in vitro. In addition, the MAPK/PI3K/AKT signaling pathway was inhibited by CME treatment in LPS-induced RAW 264.7 cells. Moreover, CME upregulated the expression of immune-related genes and enhanced the anti-infection ability of cells. Future investigations will aim to provide better understanding of the function of Chinese liquor. Indeed, both animal and human studies must be designed, as extrapolations cannot be made from cell culture studies to humans.

\section{Conflicts of interest}

The authors declare no conflict of interest.

\section{Acknowledgements}

Authors are greatly thankful to the Shenzhen City Key project of science and technology program (No. JCYJ20140418095735538) for financial support, valuable suggestions and guidelines.

\section{References}

1 D. N. Khansari and T. Gustad, Mech. Ageing Dev., 1991, 57, 87-100.

2 C. Kenyon, M. Weinstein and K. Oconnor, Reproductive Aging, 2010, vol. 1204, pp. 156-162.

3 K. H. Baek and D. Z. Skinner, J. Agric. Food Chem., 2012, 1, 34-40.

4 R. G. Alscher, J. L. Donahue and C. L. Cramer, Physiol. Plant., 1997, 100, 224-233.

5 A. S. Bess, T. L. Crocker, I. T. Ryde and J. N. Meyer, Nucleic Acids Res., 2012, 40, 7916-7931.

6 S. Kaur, S. Jobling, C. S. Jones, L. R. Noble, E. J. Routledge and A. E. Lockyer, PLoS One, 2015, 10.

7 A. T. Nguyen and A. G. Oglesby-Sherrouse, Appl. Microbiol. Biotechnol., 2016, 100, 6141-6148.

8 D. A. Garsin, J. M. Villanueva, J. Begun, D. H. Kim, C. D. Sifri, S. B. Calderwood, G. Ruvkun and F. M. Ausubel, Science, 2003, 300, 1921.

9 S. B. Flohe, S. Flohe and F. U. Schade, Innate Immun., 2008, 14, 333-344.

10 Y. H. Jing, J. L. Yan, Q. J. Wang, H. C. Chen, X. Z. Ma, J. Yin and L. P. Gao, Exp. Gerontol., 2018, 77-86.

11 A. Drexler, A. Nuss, E. Hauck, E. Glennon, K. Cheung, M. Brown and S. Luckhart, J. Exp. Biol., 2013, 216, 208-217.

12 J. E. Welke and C. A. Zini, J. Braz. Chem. Soc., 2011, 22, 609622.

13 M. Jayachandran, B. Chandrasekaran and N. Namasivayam, Eur. J. Pharmacol., 2015, 762, 102-111.

14 M. V. Baev, D. Baev, A. J. Radek and J. W. Campbell, Appl. Microbiol. Biotechnol., 2006, 71, 317-322.

15 H. Wang, X. Guo, X. Hu, T. Li, X. Fu and R. H. Liu, Food Chem., 2017, 217, 773-781.

16 H. Wang, X. Guo, J. Liu, T. Li, X. Fu and R. H. Liu, RSC Adv., 2017, 7, 28931-28939.

17 T. Schmitz, J. Ritter, S. Mueller, U. Felderhoff-Mueser, L.-J. Chew and V. Gallo, J. Neurosci., 2011, 31, 4327-4344.

18 Y. Qiao, Y. Zhao, Q. Wu, L. Sun, Q. Ruan, Y. Chen, M. Wang, J. Duan and D. Wang, PLoS One, 2014, 9, e91825.

19 C. H. Schenck, S. A. Lee, M. A. C. Bornemann and M. W. Mahowald, J. Forensic Sci., 2009, 54, 1475-1484.

20 P. Varma, N. Nisha, K. R. Dinesh, A. V. Kumar and R. Biswas, J. Mol. Microbiol. Biotechnol., 2011, 20, 137-143.

21 Y. J. Lee, N.-Y. Kim, Y.-A. Suh and C. Lee, Korean J. Physiol. Pharmacol., 2011, 15, 1-7.

22 H. Zhao, P. Gao, C. Zhang, W. Ma and Y. Jiang, J. Insect Sci., 2013, 13, 80.

23 N. Drela, Postepy Biochem., 2014, 60, 221-232.

24 R. Burstein, S. Frankel, S. D. Soule and H. T. Blumenthal, Am. J. Obstet. Gynecol., 1973, 116, 271-276.

25 K. CH, J. RG, C. YH, M. SK, K. WJ and K. GY, Toxicol. in Vitro, 2013, 27, 782-787.

26 F. Xia, C. Wang, Y. Jin, Q. Liu, Q. Meng, K. Liu and H. Sun, J. Atheroscler. Thromb., 2014, 21, 768-783.

27 X. L. Lu, C. H. Zhao, X. L. Yao and H. Zhang, Biomed. Pharmacother., 2017, 85, 658. 
28 M. Schieber and N. S. Chandel, Curr. Biol., 2014, 24, R453R462.

29 L. Di Renzo, L. T. Marsella, A. Carraro, R. Valente, P. Gualtieri, S. Gratteri, D. Tomasi, F. Gaiotti and A. De Lorenzo, Mediators Inflammation, 2015, 1-13.

30 U. Senftleben, Y. Cao, G. Xiao, F. R. Greten, G. Krähn, G. Bonizzi, Y. Chen, Y. Hu, A. Fong and S. C. Sun, Science, 2001, 293, 1495-1499.

31 G. D. Sharma, H. T. Nguyen, A. S. Antonov, R. G. Gerrity, T. von Geldern and K. N. Pandey, Mol. Cell. Biochem., 2002, 233, 165-173.

32 M. J. Mondrinos, P. A. Kennedy, M. Lyons, C. S. Deutschman and L. E. Kilpatrick, Shock, 2013, 39, 467-479.
33 B. Pavan, A. Dalpiaz, L. Marani, S. Beggiato, L. Ferraro, D. Canistro, M. Paolini, F. Vivarelli, M. C. Valerii and A. Comparone, Front. Pharmacol., 2018, 9, 467-479.

34 R. A. Baxter, J. Cosmet. Dermatol., 2010, 7, 2-7.

35 T. Wei, M. Zeng, L. I. Ke and Y. L. Wang, Food \& Machinery, 2016.

36 S. Durai, S. K. Pandian and K. Balamurugan, J. Basic Microbiol., 2011, 51, 243-252.

37 G. V. Mallo, C. L. Kurz, C. Couillault, N. Pujol, S. Granjeaud, Y. Kohara and J. J. Ewbank, Curr. Biol., 2002, 12, 1209-1214. 38 G. Jebamercy and K. Balamurugan, Microbiol. Immunol., 2012, 56, 825-835. 